\title{
Risk of Suicide Attempt after Thyroidectomy: A Nationwide Population Study in South Korea
}

\author{
Hyewon Kim¹, Yuwon Kim², Myung-Hee Shin ${ }^{3}$, Kwan Woo Choi ${ }^{4}$, Man Ki Chung ${ }^{5}$, and Hong Jin Jeon ${ }^{1,6,7} \bowtie$ \\ ${ }^{1}$ Department of Psychiatry, Depression Center, Samsung Medical Center, Sungkyunkwan University School of Medicine, Seoul, Republic of Korea \\ ${ }^{2}$ Department of Data Science, Evidnet, Seongnam, Republic of Korea \\ ${ }^{3}$ Department of Social and Preventive Medicine, Sungkyunkwan University School of Medicine, Suwon, Republic of Korea \\ ${ }^{4}$ Department of Psychiatry, Korea University College of Medicine, Seoul, Republic of Korea \\ ${ }^{5}$ Department of Otorhinolaryngology-Head and Neck Surgery, and Head and Neck Cancer Center, Samsung Medical Center, \\ Sungkyunkwan University School of Medicine, Seoul, Republic of Korea \\ ${ }^{6}$ Korean Psychological Autopsy Center (KPAC), Seoul, Republic of Korea \\ ${ }^{7}$ Department of Health Sciences \& Technology, Department of Medical Device Management \& Research, and Department of Clinical Research Design \\ \& Evaluation, Samsung Advanced Institute for Health Sciences \& Technology (SAIHST), Sungkyunkwan University, Seoul, Republic of Korea
}

Objective To investigate the association between thyroidectomy and suicide attempt.

Methods A nationwide population-based electronic medical records database of South Korea between January 1, 2009 and June 30, 2016 was used to investigate incidence rate ratios (IRRs) of suicide attempts and probable suicide attempts before and after thyroidectomy using a self-controlled case series design.

Results In 2,986 patients who attempted suicide or probable suicide, the IRRs of suicidal behaviors during risk periods one year before and after thyroidectomy were investigated. Generally, after thyroidectomy, there was no increase in IRR compared to the non-risk period. When data were analyzed according to thyroidectomy type, after partial thyroidectomy, IRR increased up to 1.43 (95\% CI: $1.03-1.98$, $\mathrm{p}=0.032$ ) in the days $91-181$ period. In the subgroup with major depressive disorder (MDD), the IRR increased up to 1.74 (95\% CI: $1.21-2.51, \mathrm{p}=0.003$ ) before thyroidectomy, and increased up to 1.67 (95\% CI: $1.16-2.41, \mathrm{p}=0.006$ ) after thyroidectomy.

Conclusion Although the general risk of suicide attempt was not increased after thyroidectomy, patients with MDD showed increased risk of suicide attempt before and after thyroidectomy. These results suggest that suicidality should be evaluated when depressive symptoms are present in patients who have undergone thyroidectomy.

Psychiatry Investig 2021;18(1):39-47

Key Words Suicide attempt, Thyroidectomy, Incidence rate ratios.

\section{INTRODUCTION}

Thyroidectomy is surgical removal of all or part of the thyroid gland and can affect secretion of thyroid hormones, causing hypothyroidism. Hypothyroidism is known to be associated

\footnotetext{
Received: May 12, 2020 Revised: October 22, 2020

Accepted: October 24, 2020

$\triangle$ Correspondence: Hong Jin Jeon, $\mathrm{MD}, \mathrm{PhD}$

Department of Psychiatry, Depression Center, Samsung Medical Center, Sungkyunkwan University School of Medicine, Seoul, Republic of Korea; Director of the Korean Psychological Autopsy Center, Seoul, Republic of Korea; Department of Health Sciences \& Technology, Department of Medical Device Management \& Research, and Department of Clinical Research Design \& Evaluation, Samsung Advanced Institute for Health Sciences \& Technology (SAIHST), Sungkyunkwan University, 81 Irwon-ro, Gangnam-gu, Seoul 06351, Republic of Korea

Tel: +82-2-3410-3586, Fax: +82-2-3410-0050, E-mail: jeonhj@skku.edu

(c) This is an Open Access article distributed under the terms of the Creative Commons Attribution Non-Commercial License (https://creativecommons.org/licenses/by$\mathrm{nc} / 4.0$ ) which permits unrestricted non-commercial use, distribution, and reproduction in any medium, provided the original work is properly cited.
}

with psychiatric manifestations such as $\operatorname{mood}^{1,2}$ and cognition; ${ }^{1,3}$ rarely, severe hypothyroidism in myxedema can lead to agitation and psychosis. ${ }^{4,5}$ In addition, studies about thyroidectomy have shown that thyroidectomy increases psychiatric morbidity. Recently, we studied the incidence of major depressive disorder (MDD) after thyroidectomy using claim data of South Korea and revealed that MDD increased between one and two years after surgery, especially for those over 50 years old. ${ }^{6}$ In addition, another study found that patients with thyroid cancer who undergo thyroidectomy have depressive disorder more frequently compared to normal controls. ${ }^{7}$ A comparative study found that depressed patient with hypothyroidism had more anxiety symptoms and greater agitation and fewer severe core depressive symptoms and biological signs of MDD, and suggested that MDD associated with hypothyroidism is differentiated from MDD without hypothyroidism. ${ }^{8}$ Another study found the increased regional homogeneity reflecting 
disturbed neural modulations in multiple brain areas in patients with hypothyroidism after total thyroidectomy, and this change was associated with poorer mental quality of life and depression. ${ }^{9}$ In an animal model, lateral habenula in the brain region is suggested to be associated with depressive symptoms that occur after thyroidectomy. ${ }^{10}$

Many studies have investigated suicide attempts, and various factors have been associated with suicide attempt, including genetic loading, ${ }^{11,12}$ brain dysfunction, ${ }^{13,14}$ psychopathologies, ${ }^{15,16}$ emotional states such as aggression and impulsivity, ${ }^{12,17}$ comorbid physical diseases including cancers, ${ }^{18,19}$ use of hypnotics or substance, ${ }^{12,20}$ and personal experience such as childhood trauma. ${ }^{11,21}$ In addition, previous studies have consistently reported that depression is the most common psychiatric disorder in people died by suicide. ${ }^{22-25}$

Thyroidectomy and suicide attempt are suggested to share many features considering that hypothyroidism is associated with psychiatric manifestations, depressive symptoms can occur after thyroidectomy, and thyroid disease such as thyroid cancer lead to thyroidectomy. However, as mentioned above, although hypothyroidism caused by thyroidectomy can lead to depression, the manifestation of it is different from that unrelated to thyroid problem. Therefore, it is an important to identify the effect of psychosocial changes after thyroidectomy on suicide attempts. However, the association between thyroidectomy and suicide attempt is not well established.

This study aimed to investigate the association between thyroidectomy and suicide attempt. In this study, we hypothesized that 1 ) thyroidectomy would be associated with suicide attempt or probable suicide attempt, 2) the risk of suicide attempts or probable suicide attempts after total thyroidectomy would be higher than after partial thyroidectomy, and 3) the risk of suicide attempts or probable suicide attempts after thyroidectomy would be higher in people with thyroid cancer than in people with benign thyroid disease.

\section{METHODS}

\section{Data source}

This study used data obtained from the Korea National Health Insurance (KNHI) Claims Database of the Health Insurance Review and Assessment Service (HIRA) in South Korea. ${ }^{26}$ HIRA is a public institution responsible for evaluation of medical expenses of medical institutions and appropriateness of medical care benefits. Such data are open to the public and cover all health services including admission, emergency room visits, ambulatory care visits, and pharmaceutical services. This data provide medical information for approximately 50 million Koreans, but does not include information related to death. Nearly $97 \%$ of the total population is enrolled in this service, while the remaining 3\% is covered by the Medical Aid Program. HIRA data are anonymized to protect the privacy of individuals, and the HIRA provides individual numbers for researches that replace the social security numbers. The data are widely used in various fields of medical research. ${ }^{20,27,28}$ The study protocol was approved by the Institutional Review Board of Samsung Medical Center (No. 2019-04-022).

\section{Case identification}

We used HIRA data from January 1, 2009 to June 30, 2016 and identified 187,176 patients who had undergone partial (P4551, P4553) or total (P4552, P4554, P4561) thyroidectomy only once during the period January 1, 2011 to June 30, 2015.

Patients having received an intentional self-harm code (X60X84) were categorized into the suicide attempt group based on the International Statistical Classification of Disease and Related Health Problems 10th revision (ICD-10). Those with several ICD-10 codes not explicitly identified as suicide attempt but highly suspected as suicide attempts were included in the group of probable suicide attempts (Supplementary Table 1 in the online-only Data Supplement). According to previous studies, it is estimated that $62-81 \%$ of suicide attempts are not detected by physicians. ${ }^{29-31}$ We assumed that because of self-concealment, many people would not have suicide attempt code even if they had come to the hospital for physical problems associated with suicidal intention. Previous studies have shown that many people tend to conceal their suicidal intention from physicians, ${ }^{29,31,32}$ and even if they come to the hospital after a suicide attempt, physicians may not notice suicidality when they focus on the physical condition that was caused by suicidal behavior. ${ }^{30,33}$ A previous study has shown that it is difficult to determine the intention of behavior in cases of death due to hanging or suffocation, poisoning, falling, and drowning. ${ }^{34}$ Similarly, when treated for these causes, suicidality is likely to be ignored. Also, even if the physician knows suicidal intention of a patient, there is a possibility that the physician enters codes only related to physical problem resulted from suicide attempt. If the outcome is defined by the codes of suicide attempt, underestimation of suicidality may occur, and this can lead to bias. Because of this, we also established a probable suicide attempt group and included them in the analysis.

Among 187,176 patients who had undergone thyroidectomy during the study period, 16 had codes of suicide attempt and 3,362 had codes of probable suicide attempts. One patient with suicide attempt and 389 patients with probable suicide attempt in 2009 were excluded. In addition, two patients were excluded because of probable suicide attempt on the day of thyroidectomy. Including four patients with both codes of suicide attempt and probable suicide attempt, 2,986 patients were in- 
cluded in statistical analysis (Supplementary Figure 1 in the online-only Data Supplement).

We performed subgroup analyses to identify patterns and outcomes according to thyroidectomy type, thyroid disease and diagnosis of MDD. For subgroup analysis according to thyroid disease, people with ICD-10 code of C73 were classified into the thyroid cancer group while those without this code were classified under the benign thyroid disease. For subgroup analysis in people with MDD, people with ICD-10 codes of F32 or F33 were classified into the MDD group.

\section{Self-controlled case series design}

We used a self-controlled case series (SCCS) study design ${ }^{35}$ to investigate the association of thyroidectomy with suicide attempts and probable suicide attempts. In this study design, individuals act as their own control. Because the comparisons were made within individuals, only those who experienced an event were included, while all time-invariant confounders were eliminated (i.e., genetic factors, individual frailty, and psychosocial factors).$^{36}$ SCCS represents an alternative epidemiologic study design to resolve difficulties in selecting an appropriate comparison group in cohort or case-control studies investigating suicide attempt. ${ }^{35}$ Considering that various complex factors and their timing affect suicide attempts, case-control design has major limitations in the research on suicide attempt due to inadequate control for key confounders such as psychiatric condition, psychosocial stress, genetic loading, and personal experience. The SCCS was determined as an appropriate model for attenuating significant bias in research on suicide attempt. ${ }^{20,37}$ The SCCS method estimates the incidence of events during risk periods compared with the incidence during all other periods. ${ }^{35}$

\section{Exposure and outcomes}

The SCCS design requires establishing the risk period likely affected by the exposure during the observation period. The index date of exposure was defined as the day of thyroidectomy. We defined the pre- and post-exposure periods as one year before and after the index date, respectively. Regarding postexposure period, because the direct evidence on risk of suicide attempt after thyroidectomy was insufficient, we established risk period considering relevant previous studies. According to a study of suicide in cancer patients, the standardized mortality ratio of suicide among patients with thyroid cancer was highest at 2 to 11 months after diagnosis. ${ }^{19}$ And other studies have reported the increased risk of depressive disorder after thyroidectomy up to postoperative year $1 .{ }^{6,7}$ Considering that depression constitutes $60-70 \%$ of all suicides, ${ }^{38}$ the risk of suicide attempt is assumed to increase when the risk of depression increases. In addition, we had established the pre-expo- sure risk period as one year before thyroidectomy considering the time from diagnosis of thyroid cancer to thyroidectomy. According to a previous study of cancer patients, distress is known to be the greatest in the waiting period for surgery or treatment after diagnosis. ${ }^{39}$ In South Korea, the waiting time from diagnosis of thyroid cancer to surgery varies depending on the individuals, but it is known to take up to $10-12$ months. ${ }^{40,41}$

We then divided the period into three-month intervals. The pre-exposure period was then divided into -365--271, -270$-181,-180--91$, and $-90^{-}-1$ segments with regard to the days before thyroidectomy. The post-exposure period was divided into 1-90, 91-180, 181-270, and 271-365 segments and represents the days after thyroidectomy. In order to set the subdivided risk period, we had examined the incidence rate ratios (IRR) in various time windows, and found there was no difference in trend of risk change in various time windows. Therefore, we divided the risk period into three-month intervals considering the general interval for visit to physicians. The first event in individuals who attempted multiple suicides or probable suicides was analyzed in this study.

\section{Statistical analysis}

To investigate the association between thyroidectomy and suicide attempts or probable suicide attempts, we investigated the IRRs of suicide attempts and probable suicide attempts during in sub-risk periods. Data from day 0 (day of thyroidectomy) were not included in the analysis. Adjusted IRRs and corresponding 95\% CIs were calculated using conditional Poisson regression after adjusting for age and season. The IRR in each sub-risk period was defined as the relative ratio of incidence of event in sub-risk period compared to that in non-risk period. The same methods were applied to subgroup analyses according to thyroidectomy type and thyroid disease and subgroup analysis in people with MDD.

\section{RESULTS}

\section{Sample characteristics}

Among 187,176 patients who had undergone thyroidectomy during the study period, excluding those who were coded with suicide attempt or probable suicide attempt in 2009 or on the day of thyroidectomy, 2,986 individuals who had record of at least one suicide attempt or probable suicide attempt per protocol were included in our analyses. Of these, 15 had a record of a suicide attempt, while 2,971 had record of a probable attempt. Four individuals had both codes of suicide attempt and probable suicide attempt. Males constituted $13.33 \%$ of those attempting suicides and $22.01 \%$ of those attempting probable suicide. The mean age of patients having undergone thyroidectomy was 47.27 (SD=15.17) years in suicide attempt- 
Table 1. Demographic and clinical characteristics of subjects in suicide attempt and probable suicide attempt groups

\begin{tabular}{|c|c|c|c|c|}
\hline \multirow{2}{*}{ Variables } & \multirow{2}{*}{$\begin{array}{l}\text { Suicide attempt }{ }^{\mathrm{a}} \\
(\mathrm{N}=15)\end{array}$} & \multicolumn{3}{|c|}{ Probable suicide attempt $\mathrm{t}^{\mathrm{b}}(\mathrm{N}=2,971)$} \\
\hline & & $\mathrm{MDD}^{\mathrm{c}}$ & Non-MDD & Total \\
\hline Male (N, \%) & $2(13.33)$ & $91(16.25)$ & $563(23.35)$ & $654(22.01)$ \\
\hline Age at thyroidectomy (SD) & $47.27(15.17)$ & $53.47(12.56)$ & $49.82(12.30)$ & $50.51(12.43)$ \\
\hline Age at first suicide or probable suicide attempt (SD) & $48.60(13.81)$ & $53.89(12.82)$ & $50.35(12.52)$ & $51.01(12.65)$ \\
\hline Diagnosis of thyroid cancerd $(\mathrm{N}, \%)$ & $13(86.67)$ & $473(86.81)$ & $2093(84.46)$ & $2566(86.37)$ \\
\hline \multicolumn{5}{|l|}{ Number of events } \\
\hline \multicolumn{5}{|l|}{ Pre-exposed risk period } \\
\hline Number of events & 0 & 86 & 327 & 413 \\
\hline Patient-days & 5,475 & 204,400 & 880,015 & $1,084,415$ \\
\hline No. of events/patient-days (per 10,000) (95\% CI) & $0(0.00-6.74)$ & $4.21(3.32-5.10)$ & $3.72(3.31-4.12)$ & $3.81(3.44-4.18)$ \\
\hline \multicolumn{5}{|l|}{ Post-exposed risk period } \\
\hline Number of events & 4 & 103 & 316 & 419 \\
\hline Patient-days & 5,400 & 201,600 & 867,960 & $1,069,560$ \\
\hline No. of events/patient-days (per 10,000) (95\% CI) & $7.41(2.02-18.96)$ & $5.11(4.12-6.10)$ & $3.64(3.24-4.04)$ & $3.92(3.54-4.29)$ \\
\hline \multicolumn{5}{|l|}{ Non-risk period } \\
\hline Number of event & 11 & 371 & 1,768 & 2,139 \\
\hline Patient-days & 27,465 & $1,025,360$ & 4,414,541 MDD & $5,439,901$ \\
\hline No. of events/patient-days (per 10,000) $(95 \%$ CI) & $4.01(2.00-7.17)$ & $3.62(3.25-3.99)$ & $4.00(3.82-4.19)$ & $3.93(3.77-4.10)$ \\
\hline
\end{tabular}

${ }^{a}$ subjects who had undergone thyroidectomy only once and had at least one suicide attempt, ${ }^{b}$ subjects who had undergone thyroidectomy only once and had at least one probable suicide attempt, ${ }^{\mathrm{c}}$ diagnosis by F32, F33 per ICD-10 before and after thyroidectomy, ${ }^{\mathrm{d}}$ diagnosis by C73 per ICD-10. MDD: major depressive disorder, SD: standard deviation, CI: confidence interval

ers and $50.51(\mathrm{SD}=12.43)$ years in probable suicide attempters. The mean age of patients at first suicide attempt was 48.60 years $(\mathrm{SD}=13.81)$, and the mean age of patients at first probable suicide attempt was 51.01 years $(\mathrm{SD}=12.65)$. About $87 \%$ of all patients were diagnosed with thyroid cancer. Numbers of events/person-days (per 10,000) or suicide attempts or probable suicide attempts during the non-risk, pre-exposed risk, and post-exposed risk periods were 4.01 (95\% CI: 2.00-7.17), 0 (no suicide attempt was occur during pre-exposed risk period), and 7.41 (95\% CI: 2.02-18.96), respectively, in the suicide attempt group and 3.93 (95\% CI: 3.77-4.10), 3.81 (95\% CI: 3.44-4.18), and 3.92 (95\% CI: 3.54-4.29) in the probable suicide attempt group. Further, we divided the probable suicide attempt group into MDD and non-MDD subgroups. In the MDD subgroup, there were fewer men, and the number of events/patient-days was higher in the risk period than in the non-risk period compared to the non-MDD group (Table 1).

\section{Incidence rate ratios of suicide attempts and probable suicide attempts before and after thyroidectomy}

Table 2 shows the IRRs of total of suicide attempt and probable suicide attempt before and after thyroidectomy in different risk windows. After adjusting for age and season, there was no statistical difference of IRRs in divided risk periods.

Table 3 shows the IRRs of suicide attempt or probable sui-
Table 2. Adjusted Incidence Rate Ratios of total of suicide attempts ${ }^{\mathrm{a}}$ and probable suicide attempts ${ }^{\mathrm{b}}$ before and after the thyroidectomy in different risk windows

\begin{tabular}{ccc}
\hline Risk period $^{\mathrm{c}}$ (days) & IRR $(95 \% \mathrm{CI})$ & p-value \\
\hline$-365--271$ & $1.05(0.87-1.26)$ & 0.624 \\
$-270--181$ & $1.09(0.90-1.31)$ & 0.388 \\
$-180--91$ & $0.82(0.66-1.01)$ & 0.068 \\
$-90--1$ & $0.92(0.75-1.13)$ & 0.419 \\
$1-90$ & $0.88(0.72-1.09)$ & 0.242 \\
$91-180$ & $1.06(0.88-1.29)$ & 0.524 \\
$181-270$ & $0.95(0.77-1.16)$ & 0.593 \\
$271-365$ & $1.10(0.91-1.32)$ & 0.337 \\
\hline
\end{tabular}

Adjusted for age and season. ${ }^{\text {a }}$ subjects who had undergone thyroidectomy and had at least one suicide attempt $(\mathrm{N}=15)$, ${ }^{\text {b subjects }}$ who had undergone thyroidectomy and had at least one probable suicide attempt $(\mathrm{N}=2,971)$, cinterval from the day of thyroidectomy. IRR: incidence rate ratio, CI: confidence interval

cide attempt before and after thyroidectomy in different risk windows according to types of thyroidectomy and thyroid disease. In the patients who had undergone partial thyroidectomy, IRR increased 91-180 days after thyroidectomy (IRR: 1.43, 95\% CI: $1.03-1.98, \mathrm{p}=0.032$ ). In other risk periods, there was no significant difference in IRR. In patients who had undergone total thyroidectomy, there was no significant difference in IRRs in divided risk periods. In the results according to thy- 
Table 3. Adjusted Incidence Rate Ratios of total of suicide attempts ${ }^{\mathrm{a}}$ and probable suicide attempts ${ }^{\mathrm{b}}$ before and after the thyroidectomy in different risk windows according to thyroidectomy type and thyroid disease

\begin{tabular}{|c|c|c|c|c|}
\hline \multirow{3}{*}{$\begin{array}{l}\text { Risk period }^{\mathrm{c}} \\
\quad \text { (days) }\end{array}$} & \multicolumn{2}{|c|}{ Thyroidectomy type } & \multicolumn{2}{|c|}{ Thyroid disease } \\
\hline & $\begin{array}{l}\text { Partial thyroidectomy } \\
\qquad(\mathrm{N}=748)\end{array}$ & $\begin{array}{l}\text { Total thyroidectomy } \\
\qquad(\mathrm{N}=2,234)\end{array}$ & $\begin{array}{l}\text { Thyroid cancerd } \\
\quad(\mathrm{N}=2,576)\end{array}$ & $\begin{array}{l}\text { Benign thyroid disease } \\
\qquad(\mathrm{N}=406)\end{array}$ \\
\hline & \multicolumn{4}{|c|}{ IRR (95\% CI) } \\
\hline$-365--271$ & $0.85(0.56-1.28)$ & $1.12(0.91-1.38)$ & $1.02(0.83-1.25)$ & $1.22(0.76-1.97)$ \\
\hline$-270--181$ & $0.84(0.55-1.28)$ & $1.17(0.95-1.45)$ & $1.13(0.92-1.37)$ & $0.84(0.47-1.50)$ \\
\hline$-180--91$ & $0.84(0.56-1.28)$ & $0.81(0.63-1.04)$ & $0.80(0.64-1.01)$ & $0.92(0.52-1.60)$ \\
\hline$-90--1$ & $0.67(0.42-1.07)$ & $1.00(0.80-1.26)$ & $0.91(0.73-1.13)$ & $1.01(0.59-1.72)$ \\
\hline $1-90$ & $1.01(0.69-1.49)$ & $0.84(0.66-1.08)$ & $0.87(0.69-1.09)$ & $1.00(0.59-1.72)$ \\
\hline $91-180$ & $1.43(1.03-1.98)^{*}$ & $0.94(0.74-1.18)$ & $1.08(0.88-1.32)$ & $0.97(0.57-1.67)$ \\
\hline $181-270$ & $0.92(0.62-1.37)$ & $0.96(0.76-1.20)$ & $0.93(0.75-1.16)$ & $1.05(0.62-1.76)$ \\
\hline $271-365$ & $0.78(0.51-1.21)$ & $1.20(0.98-1.48)$ & $1.11(0.91-1.36)$ & $0.99(0.58-1.70)$ \\
\hline
\end{tabular}

Adjusted for age and season. a subjects who had undergone thyroidectomy and had at least one suicide attempt ( $\mathrm{N}=15)$, ${ }^{\mathrm{b}}$ subjects who had un-


per ICD-10, ${ }^{*} \mathrm{p}<0.05$. IRR: incidence rate ratio, $\mathrm{CI}$ : confidence interval

roid disease, there was no increased risk of suicide attempt or probable suicide attempt compared to the non-risk period in both groups of thyroid cancer and benign thyroid disease.

When stratified by sex and age of 50 , there was no increase in IRR after thyroidectomy in any strata, but IRR increased 270181 days before thyroidectomy in female of 50 years or older (Supplementary Table 2 in the online-only Data Supplement).

\section{Incidence rate ratios of suicide attempts and probable} suicide attempts before and after thyroidectomy in patients with major depressive disorder

According to previous studies, the risk of suicide increased for about one year after diagnosis of thyroid cancer, and the risk of depression increased for one year after thyroidectomy. ${ }^{6,719}$ In addition, depression is known to be a major cause of suicide. ${ }^{38}$ To investigate the effect of thyroidectomy on suicide attempt in people with depression, we conducted subgroup analysis including patients with diagnosis of MDD. As a result, in the pre-exposed risk period, the IRR increased in the -270-181 period (IRR 1.74, 95\% CI: 1.21-2.51, $\mathrm{p}=0.003$ ) but decreased in the -181--91 period (IRR 0.50, 95\% CI: 0.25-0.97, $\mathrm{p}=0.039)$. After thyroidectomy, IRR increased to 1.67 (95\% CI: $1.16-2.41, \mathrm{p}=0.006$ ) in the $91-180$ period (Table 4 ).

Incidence rate ratios of suicide attempts and probable suicide attempts before and after thyroidectomy in patients with major depressive disorder according to types of thyroidectomy and thyroid disease

Among patients with MDD, after partial thyroidectomy, the IRR increased in 1-90 (IRR 2.05, 95\% CI: 1.03-4.08, $\mathrm{p}=0.042$ ) and $91-180$ periods (IRR $2.13,95 \%$ CI: $1.10-4.11, \mathrm{p}=0.025$ ). In total thyroidectomy patients with MDD, the pre-exposed
Table 4. Adjusted Incidence Rate Ratios of total of suicide attempts ${ }^{\mathrm{a}}$ and probable suicide attempts ${ }^{\mathrm{b}}$ before and after the thyroidectomy in different risk windows in patients with $\mathrm{MDD}^{\mathrm{c}}(\mathrm{N}=565)$

\begin{tabular}{ccc}
\hline Risk period $^{c}$ (days) & IRR $(95 \%$ CI $)$ & p-value \\
\hline$-365--271$ & $1.09(0.70-1.70)$ & 0.687 \\
$-270--181$ & $1.74(1.21-2.51)$ & 0.003 \\
$-180--91$ & $0.50(0.26-0.97)$ & 0.039 \\
$-90--1$ & $1.33(0.88-2.01)$ & 0.179 \\
$1-90$ & $1.48(1.00-2.18)$ & 0.051 \\
$91-180$ & $1.67(1.16-2.41)$ & 0.006 \\
$181-270$ & $1.42(0.95-2.12)$ & 0.083 \\
$271-365$ & $1.15(0.74-1.79)$ & 0.530 \\
\hline
\end{tabular}

asubjects who had undergone thyroidectomy only once and had at least one suicide attempt, ${ }^{\text {b }}$ subjects who had undergone thyroidectomy only once and had at least one probable suicide attempt, ' diagnosis by F32, F33 per ICD-10 before and after thyroidectomy. MDD: major depressive disorder, SD: standard deviation, CI: confidence interval

risk period, -270--181, showed an increased IRR to 1.97 (95\% CI:1.32-2.93, $\mathrm{p}=0.001)$; in the post-exposed risk period, 181270 , the IRR increased to 1.59 (95\% CI: 1.03-2.46, $\mathrm{p}=0.35$ ). In the patients with MDD and thyroid cancer, during the preexposed risk period, the IRR increased to 1.78 (95\% CI: 1.212.63, $\mathrm{p}=0.004$ ) at $-270--181$ but decreased at $-180^{-}-91$ (IRR $0.46,95 \%$ CI: $0.22-0.96, \mathrm{p}=0.040$ ). The IRR increased to 1.75 (95\% CI: $1.19-2.58, \mathrm{p}=0.005)$ at the $91-180$ period. In patients with MDD and benign thyroid disease, the IRR increased into 2.80 (95\% CI: $1.27-6.21, \mathrm{p}=0.011$ ) in the post-exposed risk period, 1-90 (Table 5). 
Table 5. Adjusted Incidence Rate Ratios of total of suicide attempts ${ }^{\mathrm{a}}$ and probable suicide attempts ${ }^{\mathrm{b}}$ before and after the thyroidectomy in different risk windows in patients with $\mathrm{MDD}^{\mathrm{c}}$

\begin{tabular}{|c|c|c|c|c|}
\hline \multirow{3}{*}{$\begin{array}{l}\text { Risk period }^{c} \\
\quad \text { (days) }\end{array}$} & \multicolumn{2}{|c|}{ Thyroidectomy type } & \multicolumn{2}{|c|}{ Thyroid disease } \\
\hline & $\begin{array}{l}\text { Partial thyroidectomy } \\
\qquad(\mathrm{N}=132)\end{array}$ & $\begin{array}{l}\text { Total thyroidectomy } \\
\qquad(\mathrm{N}=433)\end{array}$ & $\begin{array}{l}\text { Thyroid cancer }{ }^{\mathrm{d}} \\
\qquad(\mathrm{N}=478)\end{array}$ & $\begin{array}{l}\text { Benign thyroid disease } \\
\qquad(\mathrm{N}=87)\end{array}$ \\
\hline & \multicolumn{4}{|c|}{ IRR (95\% CI) } \\
\hline$-365--271$ & $0.83(0.30-2.26)$ & $1.18(0.72-1.93)$ & $0.98(0.59-1.62)$ & $1.79(0.71-4.52)$ \\
\hline$-270--181$ & $1.05(0.43-2.61)$ & $1.97(1.32-2.93)^{* * *}$ & $1.78(1.21-2.63)^{* *}$ & $1.51(0.54-4.22)$ \\
\hline$-180--91$ & $0.45(0.11-1.84)$ & $0.52(0.24-1.09)$ & $0.46(0.22-0.96)^{*}$ & $0.77(0.19-3.19)$ \\
\hline$-90^{--1}$ & $1.12(0.45-2.77)$ & $1.40(0.88-2.23)$ & $1.24(0.78-1.97)$ & $1.94(0.77-4.90)$ \\
\hline $1-90$ & $2.05(1.03-4.08)^{*}$ & $1.30(0.81-2.09)$ & $1.27(0.81-2.00)$ & $2.80(1.27-6.21)^{*}$ \\
\hline $91-180$ & $2.13(1.10-4.11)^{*}$ & $1.50(0.97-2.35)$ & $1.75(1.19-2.58)^{* *}$ & $1.18(0.37-3.80)$ \\
\hline $181-270$ & $0.91(0.33-2.48)$ & $1.59(1.03-2.46)^{*}$ & $1.46(0.96-2.24)$ & $1.20(0.37-3.86)$ \\
\hline $271-365$ & $0.45(0.11-1.82)$ & $1.38(0.87-2.19)$ & $1.09(0.67-1.78)$ & $1.60(0.58-4.44)$ \\
\hline
\end{tabular}

Adjusted for age and season. ${ }^{a}$ subjects who had undergone thyroidectomy and had at least one suicide attempt (N=15), ${ }^{\mathrm{b}}$ subjects who had undergone thyroidectomy and had at least one probable suicide attempt ( $\mathrm{N}=2,973)$, 'diagnosis by F32, F33 per ICD-10 before and after thyroidectomy, dinterval from the day of thyroidectomy, ${ }^{*} \mathrm{p} \leq 0.05,{ }^{* *} \mathrm{p} \leq 0.01,{ }^{* * *} \mathrm{p} \leq 0.001$. MDD: major depressive disorder, IRR: incidence rate ratio, CI: confidence interval

\section{DISCUSSION}

In this study, the risk of suicide attempt one year before and after thyroidectomy was not significantly different compared to the non-risk period. In the subgroup receiving partial thyroidectomy, the risk of suicide attempt increased three to six months after thyroidectomy. When the analysis was limited to those diagnosed with or treated for MDD during the risk period, an increase in risk was observed six to nine months before the operation, and a decrease in risk was noted three to six months before the operation. Risk was increased again three to six months after thyroidectomy, which was similar in subgroup analysis according to thyroidectomy and thyroid disease types, although statistical significance was variable. These findings suggest that risk of suicide attempts after thyroidectomy generally does not increase. However, in patients with MDD, increased risk of suicide attempt was consistently observed regardless of thyroidectomy and thyroid disease types.

In addition to biological factors, individual psychosocial factors are thought to be a major factor in suicide attempt. In the case of thyroidectomy, in addition to the biological changes caused by surgery, the psychological burden due to surgery and distress regarding accompanying diseases were expected. ${ }^{39}$ Nevertheless, it is noteworthy that no substantial risk of suicide attempt has been observed. In fact, more than $80 \%$ of the people in this study were diagnosed with thyroid cancer, but suicide attempts did not increase during the risk period before and after thyroidectomy. Previous studies have shown an increase in suicide deaths after cancer diagnosis. ${ }^{42}$ Further, in the case of thyroid cancer, the standardized mortality ratio after thyroidectomy was increased up to about $14 .{ }^{19}$ However, our results did not prove the temporal relationship between thyroidectomy and suicide attempt. In other words, it is difficult to say that the risk of suicide attempt increases after thyroidectomy. As an explanation for the differences in these findings, methodological differences may play a large role. This study does not compare the study individuals with the general population or the control group but rather used the SCCS method, where comparison is performed within individuals. Therefore, the confounding effect of time-invariant factors is thought to be minimal. In addition, we should consider the status of diagnosis of thyroid cancer in South Korea. In the period of case identification, there was rapid increase in the diagnosis of thyroid cancer in South Korea. As of 2012, South Korea had the highest incidence of thyroid cancer worldwide, and the agestandardized incidence of thyroid cancer in South Korea was more than 4 times of that in United States and more than 10 times of that in China and Japan. ${ }^{43-45}$ Although the increase of risk factors such as exposure to medical radiation may affect to this, ${ }^{46,47}$ experts have raised the rapid increase of medical checkups and overdiagnosis of thyroid cancer as major causes of the high incidence of thyroid cancer in South Korea. ${ }^{48,49}$ Compared to the rapid increase of incidence, the mortality of thyroid cancer in South Korea is very low and unchanged. ${ }^{45}$ Therefore, due to the relative good prognosis of thyroid cancer in South Korea, distress after diagnosis may not be large as that of other cancers, and this also may affect to psychopathology related to suicidality.

On the other hand, in the patients with MDD, the risk of suicide attempt or probable suicide attempt increased and then decreased in pre-exposure risk period, and increased again in post-exposure risk period. As an explanation for the fluctu- 
ations in risk of suicide attempts or probable suicide attempts before thyroidectomy, it is possible that distress due to diagnosis of thyroid disease and psychopathology related to manifestation of depression were mixed during this period. In this study, we defined the MDD subgroup as people with codes of MDD at least once during study period including both of risk period and non-risk period. Therefore, the severity of depression might be inconsistent with the risk period established in this study and there might be increase of suicidality irrelevant to thyroidectomy. Nevertheless, the presence of periods where the risk of suicide attempt or probable suicide attempt increases before and after thyroidectomy suggests that suicidality should be assessed if a patient has comorbid MDD. These results were consistent regardless of types of thyroidectomy and thyroid disease. To conclude that biologic changes lead to suicide attempt, the risk of suicide attempts in total thyroidectomy should be higher than in partial thyroidectomy, but the results did not support this relationship.

This study has several limitations. First, to complement the small sample size of suicide attempt codes and the poor use of suicide attempt code in the emergency room or clinic, probable suicide attempt was defined and included in the analysis. Since probable suicide attempt was defined based on ICD-10 codes, unintentional accidents might have been included. However, in our previous study, when we defined probable suicide attempts with the same codes as this study and investigated the association between prescription of hypnotic medication and suicide attempts, the pattern of risk change before and after prescription was similar to the risk change in the suicide attempt group. Second, although age and season were adjusted and subgroup analysis was conducted in the MDD subgroup, other time-varying variables such as comorbid physical disorder and psychiatric disorder might have affected our results. However, because we used the SCCS method, the bias due to time-invariant factors such as intrinsic characteristics of individuals would be small. Third, because we used claim data, clinical information associated with thyroidectomy or suicide attempt might be lacking. For example, information about level of thyroid hormone after surgery, thyroid hormone replacement, or adequacy of postoperative treatment was not included in the analysis. Since thyroid hormone is not naturally produced after total thyroidectomy, synthetic levothyroxine $\left(\mathrm{L}-\mathrm{T}_{4}\right)$ is frequently administered. However, this study did not include such information. Fourth, because the data source does not include information related to death, it was not able to identify the death resulted from the suicide attempt or probable suicide attempt. However, because we included only the first suicide attempt or probable suicide attempt of individuals in the analyses, the lack of information related to death would not affect the results.
To our knowledge, this is the first study investigated the association between thyroidectomy and suicide attempt. This study has strength by employing real world data to identify the risk of suicide attempt in risk period before and after thyroidectomy. In addition, by including not only suicide attempt codes, but also several codes not explicitly identified as suicide attempt but highly suspected as suicide attempt, we tried to overcome the underdetection of suicide attempts at emergency rooms or clinics and provide an evidence for clinical practice.

In conclusion, while the general risk of suicide attempt was not increased after thyroidectomy, patients with MDD showed increased risk of suicide attempt after thyroidectomy. Although this study has the limitation of lacking clinical information, as real world data, this study suggests that suicidality should be evaluated when depressive symptom are present in patients who have undergone thyroidectomy.

\section{Supplementary Materials}

The online-only Data Supplement is available with this article at https://doi.org/10.30773/pi.2020.0174.

\section{Acknowledgments}

This work was supported by the Original Technology Research Program for Brain Science through the National Research Foundation of Korea (NRF) funded by the Ministry of Science and ICT (No. NRF-2016M3C7A1947307; PI HJJ), and by the Bio \& Medical Technology Development Program of the NRF funded by the Korean government, MSIP (No. NRF-2017M3A9F1027323; PI HJJ).

\section{Conflicts of Interest}

The authors have no potential conflicts of interest to disclose.

\section{Author Contributions}

Conceptualization: Hyewon Kim, Man Ki Chung, Hong Jin Jeon. Data curation: Hyewon Kim. Formal analysis: Yuwon Kim, Myung-Hee Shin. Funding acquisition: Hong Jin Jeon. Investigation: Hyewon Kim. Methodology: Hyewon Kim, Yuwon Kim. Project administration: Hong Jin Jeon. Supervision: Hong Jin Jeon. Writing-original draft: Hyewon Kim. Writing_review \& editing: Hyewon Kim, Kwan Woo Choi, Man Ki Chun, Hong Jin Jeon.

\section{ORCID iDs}

Hyewon Kim

Yuwon Kim

Myung-Hee Shin

Kwan Woo Choi

Man Ki Chung

Hong Jin Jeon

https://orcid.org/0000-0001-5665-8280

https://orcid.org/0000-0002-0747-7376

https://orcid.org/0000-0002-0509-286X

https://orcid.org/0000-0002-0854-3507

https://orcid.org/0000-0002-1435-7786

https://orcid.org/0000-0002-6126-542X

\section{REFERENCES}

1. Constant EL, Adam S, Seron X, Bruyer R, Seghers A, Daumerie C. Anxiety and depression, attention, and executive functions in hypothyroidism. J Int Neuropsychol Soc 2005;11:535-544.

2. Davis JD, Tremont G. Neuropsychiatric aspects of hypothyroidism and treatment reversibility. Minerva Endocrinol 2007;32:49-65.

3. Correia N, Mullally S, Cooke G, Tun TK, Phelan N, Feeney J, et al. Evidence for a specific defect in hippocampal memory in overt and subclinical hypothyroidism. J Clin Endocrinol Metab 2009;94:3789-3797. 
4. Mavroson MM, Patel N, Akker E. Myxedema Psychosis in a Patient With Undiagnosed Hashimoto Thyroiditis. J Am Osteopath Assoc 2017;117:50-54.

5. Parikh N, Sharma P, Parmar C. A case report on myxedema madness: curable psychosis. Indian J Psychol Med 2014;36:80-81.

6. Choi KW, Kim Y, Fava M, Mischoulon D, Na EJ, Kim SW, et al. Increased morbidity of major depressive disorder after thyroidectomy: a nationwide population-based study in South Korea. Thyroid 2019;29: 1713-1722.

7. Choi HG, Park B, Ji YB, Tae K, Song CM. Depressive disorder in thyroid cancer patients after thyroidectomy: a longitudinal follow-up study using a national cohort. Otolaryngol Head Neck Surg 2019;160: 239-245.

8. Mowla A, Kalantarhormozi MR, Khazraee S. Clinical characteristics of patients with major depressive disorder with and without hypothyroidism: a comparative study. J Psychiatr Pract 2011;17:67-71.

9. Shin YW, Choi YM, Kim HS, Kim DJ, Jo HJ, O’Donnell BF, et al. Diminished quality of life and increased brain functional connectivity in patients with hypothyroidism after total thyroidectomy. Thyroid 2016; 26:641-649.

10. Zhang Q, Feng JJ, Yang S, Liu XF, Li JC, Zhao H. Lateral habenula as a link between thyroid and serotoninergic system modiates depressive symptoms in hypothyroidism rats. Brain Res Bull 2016;124:198-205.

11. Mann JJ. Neurobiology of suicidal behaviour. Nat Rev Neurosci 2003; 4:819-828

12. Turecki G. The molecular bases of the suicidal brain. Nat Rev Neurosci 2014;15:802-816.

13. Kim K, Kim SW, Myung W, Han CE, Fava M, Mischoulon D, et al. Reduced orbitofrontal-thalamic functional connectivity related to suicidal ideation in patients with major depressive disorder. Sci Rep 2017;7: 15772.

14. Valdizan EM, Diez-Alarcia R, Gonzalez-Maeso J, Pilar-Cuellar F, Garcia-Sevilla JA, Meana JJ, et al. alpha(2)-Adrenoceptor functionality in postmortem frontal cortex of depressed suicide victims. Biol Psychiatry 2010;68:869-872.

15. Jeon HJ, Park JI, Fava M, Mischoulon D, Sohn JH, Seong S, et al. Feelings of worthlessness, traumatic experience, and their comorbidity in relation to lifetime suicide attempt in community adults with major depressive disorder. J Affect Disord 2014;166:206-212.

16. Kim H, Choi KW, Na EJ, Hong JP, Fava M, Mischoulon D, et al. Anxiety attacks with or without life-threatening situations, major depressive disorder, and suicide attempt: a nationwide community sample of Korean adults. Psychiatry Res 2018;270:257-263.

17. Mann JJ, Waternaux C, Haas GL, Malone KM. Toward a clinical model of suicidal behavior in psychiatric patients. Am J Psychiatry 1999;156: 181-189.

18. Hawton K, van Heeringen K. Suicide. Lancet 2009;373:1372-1381.

19. Zaorsky NG, Zhang Y, Tuanquin L, Bluethmann SM, Park HS, Chinchilli VM. Suicide among cancer patients. Nat Commun 2019;10:207.

20. Kim H, Kim Y, Myung W, Fava M, Mischoulon D, Lee U, et al. Risks of suicide attempts after prescription of zolpidem in people with depression: a nationwide population study in South Korea. Sleep 2020;43: zsz235.

21. Jeon HJ, Roh MS, Kim KH, Lee JR, Lee D, Yoon SC, et al. Early trauma and lifetime suicidal behavior in a nationwide sample of Korean medical students. J Affect Disord 2009;119:210-214.

22. Rich CL, Young D, Fowler RC. San Diego suicide study: I. Young vs old subjects. Arch Gen Psychiatry 1986;43:577-582.

23. Conwell Y, Duberstein PR, Cox C, Herrmann JH, Forbes NT, Caine ED. Relationship of age and Axis I diagnoses in victims of completed suicide: a psychological autopsy study. Am J Psychiatry 1996;153: 1001-1008.

24. Harwood D, Hawton K, Hope T, Jacoby R. Psychiatric disorder and personality factors associated with suicide in older people: a descriptive and case-control study. Int J Geriatr Psychiatry 2001;16:155-165.
25. Henriksson MM, Aro HM, Marttunen MJ, Heikkinen ME, Isometsa E, Kuoppasalmi KI, et al. Mental disorders and comorbidity in suicide. Am J Psychiatry 1993;150:935-940.

26. Seong SC, Kim YY, Khang YH, Heon Park J, Kang HJ, Lee H, et al. Data resource profile: the National health information database of the National health insurance service in South Korea. Int J Epidemiol 2017;46:799-800.

27. Jung SM, Kim CT, Kang EW, Kim KH, Lee S, Oh HJ, et al. Dementia is a risk factor for major adverse cardiac and cerebrovascular events in elderly Korean patients initiating hemodialysis: a Korean national population-based study. BMC Nephrol 2017;18:128.

28. Kang GW, Jung KH, Lee YS, Kim HJ, Yoon DY, Lee SH, et al. Incidence, prevalence, mortality and causes of death in systemic sclerosis in Korea: a nationwide population-based study. Br J Dermatol 2018;178:e37e39.

29. Busch KA, Fawcett J, Jacobs DG. Clinical correlates of inpatient suicide. J Clin Psychiatry 2003;64:14-19.

30. Claassen CA, Larkin GL. Occult suicidality in an emergency department population. Br J Psychiatry 2005;186:352-353.

31. Waern M, Beskow J, Runeson B, Skoog I. Suicidal feelings in the last year of life in elderly people who commit suicide. Lancet 1999;354: 917-918.

32. Friedlander A, Nazem S, Fiske A, Nadorff MR, Smith MD. Self-concealment and suicidal behaviors. Suicide Life Threat Behav 2012;42: 332-340.

33. Ilgen MA, Walton MA, Cunningham RM, Barry KL, Chermack ST, De Chavez P, et al. Recent suicidal ideation among patients in an inner city emergency department. Suicide Life Threat Behav 2009;39:508517.

34. Chan CH, Caine ED, Chang SS, Lee WJ, Cha ES, Yip PS. The impact of improving suicide death classification in South Korea: a comparison with Japan and Hong Kong. PLoS One 2015;10:e0125730.

35. Petersen I, Douglas I, Whitaker H. Self controlled case series methods: an alternative to standard epidemiological study designs. BMJ 2016; 354:i4515.

36. Whitaker HJ, Farrington CP, Spiessens B, Musonda P. Tutorial in biostatistics: the self-controlled case series method. Stat Med 2006;25: 1768-1797.

37. Man KKC, Coghill D, Chan EW, Lau WCY, Hollis C, Liddle E, et al. Association of risk of suicide attempts with methylphenidate treatment. JAMA Psychiatry 2017;74:1048-1055.

38. Sadock BJ, Sadock VA, Ruiz P. Synopsis of Psychiatry: Behavioral Sciences, Clinical Psychiatry. Philadelphia: Wolters Kluwer; 2015.

39. Northouse LL. The impact of breast cancer on patients and husbands. Cancer Nurs 1989;12:276-284

40. Kim KW. Rapid increase of thyroid cancer patients...10 months waiting time for surgery. Available at: https://news.naver.com $/ \mathrm{main} / \mathrm{read}$. nhn? mode $=$ LSD \&mid $=$ sec\&sid $1=001$ \&oid $=001$ \&aid $=0001556713$. Accessed January 11, 2021.

41. Lee JH. Early screening for thyroid cancer is 'waking sleeping dogs'. Available at: https://www.donga.com/news/article/ all/20100816/30529839/1. Accessed January 11, 2021.

42. Henson KE, Brock R, Charnock J, Wickramasinghe B, Will O, Pitman A. Risk of suicide after cancer diagnosis in England. JAMA Psychiatry 2019;76:51-60.

43. Ferlay J, Soerjomataram I, Dikshit R, Eser S, Mathers C, Rebelo M, et al. Cancer incidence and mortality worldwide: sources, methods and major patterns in GLOBOCAN 2012. Int J Cancer 2015;136:E359-E386.

44. Jung KW, Won YJ, Oh CM, Kong HJ, Lee DH, Lee KH, et al. Cancer statistics in Korea: incidence, mortality, survival, and prevalence in 2014. Cancer Res Treat 2017;49:292-305.

45. Lee SY. Thyroid cancer screening. J Korean Med Assoc 2015;58:684687.

46. Chen AY, Jemal A, Ward EM. Increasing incidence of differentiated thyroid cancer in the United States, 1988-2005. Cancer 2009;115:3801- 
3807.

47. Pellegriti G, Frasca F, Regalbuto C, Squatrito S, Vigneri R. Worldwide increasing incidence of thyroid cancer: update on epidemiology and risk factors. J Cancer Epidemiol 2013;2013:965212.

48. Ahn HS, Kim HJ, Welch HG. Korea's thyroid-cancer "epidemic"- screening and overdiagnosis. N Engl J Med 2014;371:1765-1767.

49. Brito JP, Morris JC, Montori VM. Thyroid cancer: zealous imaging has increased detection and treatment of low risk tumours. BMJ 2013; 347:f4706. 
Supplementary Table 1. Definition of suicide attempts and probable suicide attempts by International Statistical Classification of Diseases and Related Health Problems 10th revision (ICD-10)

\begin{tabular}{ll}
\hline \multicolumn{1}{c}{ Code } & \multicolumn{1}{c}{ Definition } \\
Suicide attempts & \\
X60-X84 & Intentional self-harm \\
Probable suicide & attempts \\
S61.9 & Wrist laceration \\
T43 & Poisoning by psychotropic drugs, NEC \\
T50.9 & Acute drug intoxication \\
T52 & Toxic effect of organic solvents \\
T54 & Toxic effect of corrosive substance \\
T58 & Toxic effect of carbon monoxide \\
T60 & Toxic effect of pesticides \\
T65.8 & Toxic effect of other specified substances \\
T65.9 & Toxic effect of unspecified substances \\
W32 & Handgun discharge \\
W33 & Rifle, shotgun and larger firearm discharge \\
W34 & Discharge from other and unspecified firearms \\
W75 & Accidental suffocation and strangulation in bed \\
W76 & Other accidental hanging and strangulation \\
Y10-Y34 & Event of undetermined intent \\
R09.0 & Asphyxia \\
W13-W19 & Falls \\
\hline
\end{tabular}




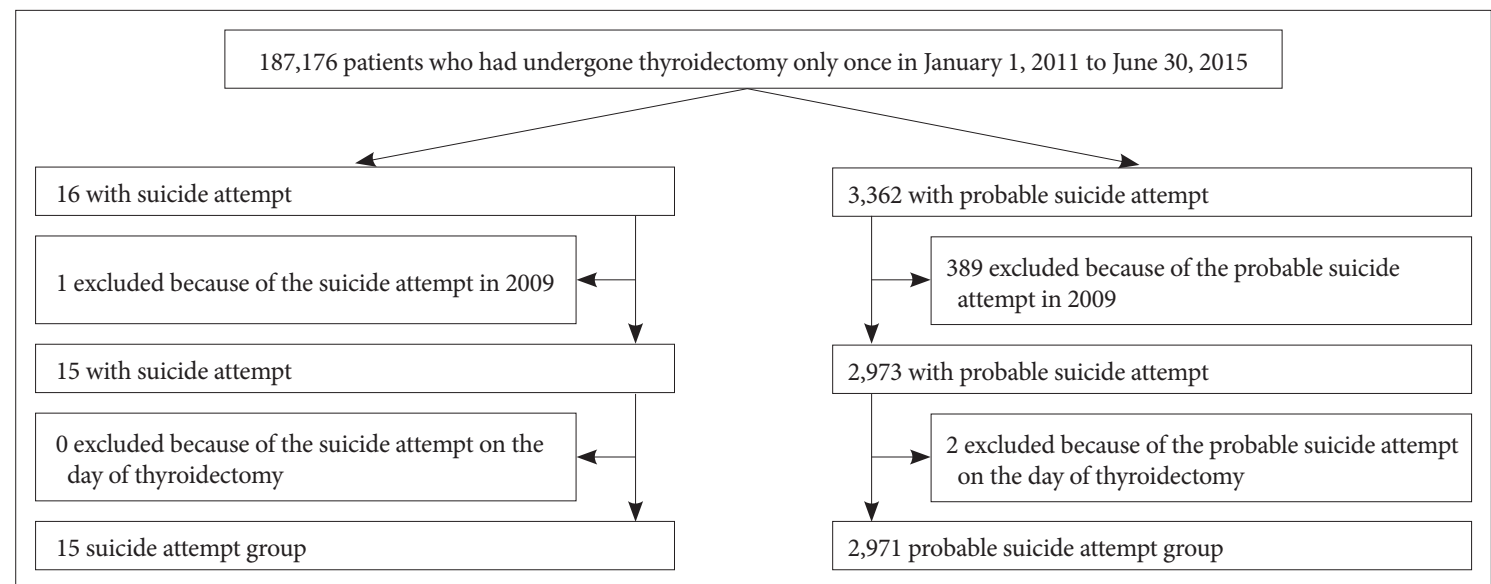

Supplementary Figure 1. Flowchart showing the identification of subjects. 
Supplementary Table 2. Adjusted Incidence Rate Ratios of total of suicide attempts ${ }^{\mathrm{a}}$ and probable suicide attempts ${ }^{\mathrm{b}}$ before and after the thyroidectomy in different risk windows stratified by sex and age

\begin{tabular}{|c|c|c|c|c|c|c|c|c|}
\hline \multirow{3}{*}{$\begin{array}{l}\text { Risk period }^{c} \\
\quad \text { (days) }\end{array}$} & \multicolumn{4}{|c|}{ Male subjects } & \multicolumn{4}{|c|}{ Female subjects } \\
\hline & \multicolumn{2}{|c|}{ Age $<50$ years $(\mathrm{N}=286)$} & \multicolumn{2}{|c|}{ Age $\geq 50$ years $(\mathrm{N}=369)$} & \multicolumn{2}{|c|}{ Age $<50$ years $(\mathrm{N}=1,063)$} & \multicolumn{2}{|c|}{ Age $\geq 50$ years $(\mathrm{N}=1,264)$} \\
\hline & $\operatorname{IRR}(95 \% \mathrm{CI})$ & $\mathrm{p}$-value & IRR (95\% CI) & p-value & $\operatorname{IRR}(95 \% \mathrm{CI})$ & $\mathrm{p}$-value & IRR (95\% CI) & $\mathrm{p}$-value \\
\hline$-365^{--271}$ & $1.05(0.57-1.93)$ & 0.886 & $1.09(0.66-1.80)$ & 0.748 & $1.28(0.96-1.71)$ & 0.092 & $0.84(0.61-1.16)$ & 0.281 \\
\hline$-270--181$ & $0.76(0.37-1.54)$ & 0.446 & $0.72(0.38-1.35)$ & 0.301 & $1.02(0.74-1.42)$ & 0.898 & $1.32(1.01-1.72)$ & 0.039 \\
\hline$-180--91$ & $0.88(0.45-1.72)$ & 0.708 & $0.71(0.38-1.34)$ & 0.294 & $0.97(0.69-1.36)$ & 0.862 & $0.70(0.49-1.01)$ & 0.055 \\
\hline$-90--1$ & $0.88(0.45-1.72)$ & 0.710 & $0.49(0.23-1.05)$ & 0.066 & $0.89(0.63-1.27)$ & 0.530 & $1.08(0.80-1.44)$ & 0.624 \\
\hline $1-90$ & $0.69(0.32-1.46)$ & 0.328 & $0.78(0.43-1.43)$ & 0.419 & $0.89(0.63-1.27)$ & 0.522 & $0.95(0.70-1.29)$ & 0.735 \\
\hline $91-180$ & $1.21(0.69-2.13)$ & 0.499 & $1.14(0.69-1.89)$ & 0.609 & $0.99(0.71-1.38)$ & 0.970 & $1.05(0.78-1.41)$ & 0.754 \\
\hline $181-270$ & $0.77(0.38-1.56)$ & 0.470 & $0.57(0.28-1.15)$ & 0.118 & $1.08(0.78-1.48)$ & 0.649 & $0.99(0.73-1.35)$ & 0.965 \\
\hline $271-365$ & $1.06(0.58-1.94)$ & 0.855 & $0.92(0.53-1.61)$ & 0.770 & $1.11(0.81-1.52)$ & 0.516 & $1.14(0.86-1.52)$ & 0.370 \\
\hline
\end{tabular}


thyroidectomy and had at least one probable suicide attempt, interval from the day of thyroidectomy. MDD: major depressive disorder, IRR: incidence rate ratio, $\mathrm{CI}$ : confidence interval 